\title{
Tree Foliages Fed to Dairy Animals in Mizoram: Traditional Medicinal Uses, Screening and Quantification of Phytochemicals
}

\author{
Rajat Buragohain* \\ Department of Animal Nutrition, College of Veterinary Sciences and Animal Husbandry, Central \\ Agricultural University, Selesih, Aizawl, Mizoram - 796014, India \\ *Corresponding author
}

\begin{abstract}
Keywords
Tree foliage, Phytochemical, Dairy animal, Mizoram.

Article Info

Accepted:

04 September 2017

Available Online: (namely, Tlangnuam, Thingsulthiah and Aibawk). Aqueous extracts of representative leaf samples were made for phytochemical screening and quantification by following different procedures. The study revealed usages of 41 different tree foliages out of which 21 were evergreen. Qualitative screening revealed 33 and 25 species positive for polyphenols and flavonoids, all for terpenoids; 22, 13 and 5 species for saponins, alkaloids and reducing sugar, respectively and 4, 10 and 13 tree species positive for glycosides, quinone and phlobatanins, respectively. DM\% of the tree leaves varied from $16.04-22.95 \%$. Total phenolics ranged from $4.3048 \pm 0.79 \%$ to $61.5564 \pm 3.91 \%$ and CT levels from $0.1165 \pm 0.04 \%$ to $7.0692 \pm 2.53 \%$ on DM basis. The flavonoids ranged from $0.0500 \pm 0.00$ to $178.4833 \pm 0.83 \mathrm{mg} \mathrm{RE} / \mathrm{g}$ dry sample and saponins varied from $10.0667 \pm 0.33$ to $112.5033 \pm 2.15 \mathrm{mg} \mathrm{DE} / \mathrm{g}$ dry sample. The alkaloids content was $0.18 \pm 0.01 \mathrm{mg} / \mathrm{g}$ in Cordia dichotoma G. Forst (Muk-fang) to $7.28 \pm 0.01 \mathrm{mg} / \mathrm{g}$ in Anogeissus acuminate (Roxb. Ex DC) Gull. (Zai-rum). Some tree species were found to utilize by the Mizo communities for treating various ailments. Baseline data about concentration of phytochemicals of tree foliages fed to dairy animal in Mizoram will help in exploiting them as herbs and potential feeds in TMR of dairy animals in Mizoram.
\end{abstract}

A B S T R A C T

The study was to identify tree foliages fed to dairy animals in Mizoram, to screen and quantify the phytochemicals and to survey traditional medicinal uses. Surveys conducted on 45 dairy farmers of 15 randomly selected villages of RDBs of Aizawl district, Mizoram

\section{Introduction}

The beneficial effects of tree leaves to livestock in addition to their nutritional values are attributed by their phytochemical constituents (Dinda et al., 2007; Podsedek, 2007). Phytochemicals of plants are responsible for numerous beneficial biological effects including antioxidant, antiinflammatory, antimicrobial and anti-cancer activities. Tree leaves provide strong protective effects against diseases associated with oxidative damage (Kaur and Kapoor,
2002) for antioxidant property (Uusiku et al., 2010). Flavonoids act as free radical scavengers and saponins present in tree leaves are known to have hypolipidemic and anticancer activity. Antioxidants of tree leaves also play pivotal role in prevention of chronic and degenerative diseases of animals. Mizoram, one of the $8^{\text {th }}$ North Eastern states of India, is situated between $21^{\circ} 58^{\prime} \mathrm{N}$ to $24^{\circ} 35^{\prime} \mathrm{N}$ latitude and $92^{\circ} 15^{\prime} \mathrm{E}$ to $93^{\circ} 29^{\prime} \mathrm{E}$ longitude spreading over 21,087 sq.km 
geographical area with 10, 91, 014 human population. The dairy farming plays important role on economic sustainability of the rural farming communities in Mizoram. Amongst livestock, dairy farming is the most popular economic activity next to piggery among the 'Mizo people' and constitutes $9.62 \%$ of total livestock population in the state (Economic Survey of Mizoram, 2013-14).

Balanced feeding is one of the biggest constraints for the dairy farmers of the state due to shortage of nutritional feeds for deficit production of grains in the state. More than $90 \%$ of the livestock feed ingredients come from other states of the mainland.

The nutritional demands of the dairy animals are met mainly by the crop residues, natural grasses, tree leaves and shrubs (Kumaresan et al., 2010) under rural production system. The tree leaves are the main components of roughage source provided to the dairy animals. The tree leaves become the sole roughage during lean season, particularly during the winter months of the year for scarcity of jungle grasses and forages in the state. A few studies had been conducted to identify the commonly utilized tree leaves for dairy animals and their nutritional composition (Das et al., 2006; Sarma et al., 2007; Samanta et al., 2009); however, no information is available about the phytochemical constituents and anti-oxidant property of the tree leaves. In the present study, therefore, an attempt was made for screening and quantification of phytochemicals and to survey about traditional medicinal uses of the tree foliages fed to dairy animals in Mizoram, India.

\section{Materials and Methods}

\section{Location of the study}

The study was conducted covering three Rural Development Blocks (RDB) of Aizawl district of Mizoram, India namely, Tlangnuam, Thingsulthiah and Aibawk and were purposively selected considering proportional dairy cattle and buffalo population of the district. Five villages were selected from each RDB, and three dairy farmers from each village were randomly selected for the study (Table 1).

\section{Sampling method and sample preparation for analysis}

Information about utilization of tree leaves for feeding dairy animals was collected from the selected farmers of each RDB. All farmers were visited and questioned about the feeding practices and utilization of tree leaves for feeding to their dairy animals.

The 'Mizo' names of the tree species were collected from farmers and the botanical names were confirmed in collaboration with the Department of Forestry, Mizoram University, Aizawl, Mizoram and referring to available literatures of Botanical Survey of India, Kolkata, India.

The healthy leaves representing different developmental stages were collected from the farmers. The leaves of each species were collected separately from all the selected farmers. After collection, leaves of the same tree species of a particular RDB were pooled together and six representative samples were drawn for analysis.

The samples of leaves were air dried under the shade and made in mesh with powdery consistency for preparation of aqueous extracts. Aqueous extracts were made utilizing about $30 \mathrm{gm}$ of dried leaves powder in Soxhlet extraction system. The extraction was performed for $48 \mathrm{hrs}$ and then the extract was concentrated by slow evaporation process through incubation at $37^{\circ} \mathrm{C}$. The yield was recorded and stored at $-20^{\circ} \mathrm{C}$ before analytical use. 
Procedures adopted for phytochemical screening

The flavonoids, polyphenols and alkaloids were analysed qualitatively following procedures outlined by Akenga et al., (2005). The saponin, anthraquinones and the presence of tannins were screened according to methods described by Sofowora (1982), Trease and Evans (1989) and Edeoga et al., (2005), respectively.

Methods for quantitative estimation of phytochemicals

The total phenolics of tree leaves as tannic acid equivalent (TAE) were estimated as per method of Makkar et al., (1993). The condensed tannins (\% as leucocyanidin equivalent) were estimated following method of Porter et al., (1986). The total flavonoid and saponin content of the extracts were determined as per methods described by Nabavi et al., (2008) and Makkar et al., (2007), respectively.

\section{Results and discussion}

\section{Description of the tree fodder species} identified

Tree leaves of forty one (41) different species were found to be utilised for feeding dairy animals in Mizoram (Table 2). Das et al., (2006) analysed leaves of twelve tree species fed to dairy cattle and found that leaves of Trema orientalis, Bauhinia variegata and Bhuhinia vahlii were rich source of protein and the leaves of Cinnamomum tamala, Bauhinia vahlii and Macaranga peltata were rich source of energy and the leaves of Trema orientalis, Bauhinia variegata and Hevea brasiliensis were rich source of calcium. Samanta et al., (2009) reported about twelve tree species and Sarma et al., (2007) described 14 different forage species utilised by the Mizo farmers for feeding dairy animals.
However, in the present study, the personal interview with the dairy farmers revealed that they utilised tree leaves of forty one different species throughout the year, out of which 21 tree species were evergreen and the rest were either large or small deciduous trees.

\section{Phytochemical screening}

The results of the qualitative screening for polyphenols, flavonoids and terpenoids in aqueous leaves extracts of the tree leaves revealed 33 and 25 species positive for polyphenols and flavonoids and all for terpenoids. Diwan et al., (2014) also reported the presence of alkaloids, flavonoids, simple phenpolics, steroids and saponins in Ficus benghalensis Linn. root extracts. Lalawmpuii et al., (2014) indicated the presence of polyphenols and flavonoids in Helicia nilagiricia Bedd., an ethnomedicinal plant of Mizoram. For saponins, alkaloids and reducing sugar, 22, 13 and 5 species were found positive, respectively.

The results thus indicated that the tree fodders are not only good sources of polyphenols, flavonoids and terpenoids, but also rich in saponins, alkaloids and reducing sugar which ensures health benefits besides providing valuable nutrients to the animals. Diwan et al., (2014) reported the presence of saponins and alkaloids in Bung (Ficus benghalensis Linn.). Sowjanya et al., (2013) indicated the presence of alkaloids and glycosides in Hmuipui/ Len-hmui [Syzygium cumini (L.) Skeels].

Out of the total 41 tree species, only 4 tree species were found positive for glycosides, 10 for quinone and 13 species for phlobatanins. Ganatra et al., (2012) reported the presence of quinine and glycosides in Ficus racemosa Linn. Egharevba and Kunle (2010) in their study indicated the presence of glycosides but absence of phlobatannins in leaves extract of Piliostigma thionningii (Schumach) commonly utilised under traditionally as 
remedy for fever, wounds, ulcers, phlobatannins were also reported by gastric/heart pain and gingivitis. Similarly, the Lalawmpuii et al., (2014) in edible leaves of presence of glycosides, quinine and various tree species.

Table.1 Villages selected for the study

\begin{tabular}{|c|c|c|}
\hline Tlanguam RDB & Thingsulthiah RDB & Aibawk RDB \\
\hline Aizawl (NT) & Seling & Aibawk \\
\hline Bawngkawn & Sesawng & Sateek \\
\hline Muthi & Saitual & Thingdawl \\
\hline Serkhan & Darlawng & Falkawn \\
\hline Sihphir & Edenthar & Thiak \\
\hline
\end{tabular}

Table.2 Different tree leaves species identified to be utilised for feeding dairy animals

\begin{tabular}{|c|c|c|}
\hline Mizo/English name & Botanical name & Description \\
\hline $\begin{array}{l}\text { An-ku/ An-ku-hawng-tial } \\
\text { (Eastern Nettle Tree) }\end{array}$ & Celtis tetrandra Roxb. & $\begin{array}{l}\text { Large deciduous, found up to } 1,100 \mathrm{~m} \text { altitude. } \\
\text { Leaves are used as fodder, fruits are prescribed for } \\
\text { remedy of amenorrhoea and colic. }\end{array}$ \\
\hline Bel-phuar (Charcoal tree) & $\begin{array}{l}\text { Trema orientalis (L.) } \\
\text { Blume. }\end{array}$ & $\begin{array}{l}\text { Evergreen tree, fast-growing and short lived, } \\
\text { leaves are fodder and plant juice is believed to be a } \\
\text { useful remedy for epilepsy. }\end{array}$ \\
\hline Bung (Banyan tree) & $\begin{array}{l}\text { Ficus } \quad \text { benghalensis } \\
\text { Linn. }\end{array}$ & $\begin{array}{l}\text { Evergreen tree, grown up to } 1,200 \mathrm{~m} \text { above MSL, } \\
\text { eaves are good fodders, Infusion of bark is used for } \\
\text { diabetes, nervous disorder, leucorrhoea, diarrhoea, } \\
\text { dysentery, and juice of stem is applied externally } \\
\text { for rheumatism, lumbago, toothache and bruises. }\end{array}$ \\
\hline Ba-tling & $\begin{array}{l}\text { Wendlandia } \\
\text { budleioides Wall. Ex } \\
\text { Wight \& Arn. }\end{array}$ & $\begin{array}{l}\text { Small evergreen tree ascending to about } 1,500 \mathrm{~m} \\
\text { above MSL, leaves are used as fodders. }\end{array}$ \\
\hline $\begin{array}{l}\text { Bil (Murtenga) - Indian Red } \\
\text { Pear }\end{array}$ & $\begin{array}{lrr}\text { Protium } & \text { serratum } \\
\text { (Wall. ex } & \text { Colebr.) } \\
\text { Engl. } & & \\
\end{array}$ & $\begin{array}{l}\text { Medium-sized or large evergreen tree, fruits are } \\
\text { eaten by the wild animals and leaves are very useful } \\
\text { for feeding to cattle and buffaloes. }\end{array}$ \\
\hline Chawm-zil (Wild Privet) & $\begin{array}{l}\text { Ligustrum robustum } \\
\text { (Roxb.) Blume }\end{array}$ & $\begin{array}{l}\text { Medium sized evergreen tree, found between } \\
\text { altitudes } 700-1800 \mathrm{~m} \text { above MSL, leaves are lopped } \\
\text { for fodders. }\end{array}$ \\
\hline Chhawn-tual & 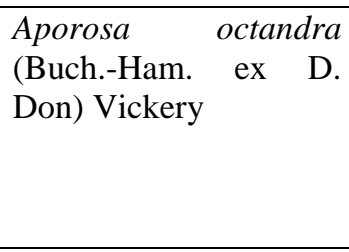 & $\begin{array}{l}\text { Small to medium sized evergreen tree, found } \\
\text { within } 900 \mathrm{~m} \text { above MSL, besides leaves as } \\
\text { roughage, bark is boiled in water and the water is } \\
\text { taken as remedy for stomach ulcer, diarrhoea and } \\
\text { dysentery, tender leaves as remedy for stomach } \\
\text { trouble. }\end{array}$ \\
\hline Hnum & $\begin{array}{l}\text { Engelhardtia spicata } \\
\text { Lechen ex Blume }\end{array}$ & $\begin{array}{l}\text { Large deciduous tree found up to } 1800 \mathrm{~m} \text { above } \\
\text { MSL, leaves are used as cattle fodder. }\end{array}$ \\
\hline Hnah-thap & $\begin{array}{l}\text { Colona floribunda } \\
\text { (Kurz) Craib }\end{array}$ & $\begin{array}{l}\text { Medium-sized deciduous tree found below } 1000 \mathrm{~m} \\
\text { above MSL, leaves are used for feeding cattle and } \\
\text { buffaloes by the dairy farmers in Mizoram. }\end{array}$ \\
\hline Hnahkiah & $\begin{array}{l}\text { Callicarpa } \\
\text { Roxb. }\end{array}$ & $\begin{array}{l}\text { Medium sized evergreen tree ascending to about } \\
1300 \mathrm{~m} \text { above MSL, leaves are lopped for fodder, } \\
\text { decoction of bark and leaves are presented for } \\
\text { diabetes, cholera, dysentery and diarrhoea, internal } \\
\text { bleeding, colic and stomach ulcer, bark juice is }\end{array}$ \\
\hline
\end{tabular}




\begin{tabular}{|c|c|c|}
\hline & & applied to freshly cut wounds. \\
\hline Hriang (Alder birch/betula) & $\begin{array}{l}\text { Betula alnoides Buch.- } \\
\text { Ham. ex D. Don. }\end{array}$ & $\begin{array}{l}\text { Medium to large sized deciduous tree found in } \\
\text { altitude } 900-1500 \mathrm{~m} \text {, leaves are lopped for feeding } \\
\text { to cattle, buffaloes, goat, wood of the plant is good } \\
\text { for furniture, tool making, used as snake-bite } \\
\text { remedy. }\end{array}$ \\
\hline $\begin{array}{l}\text { Hmui-pui/ Len-hmui (Black } \\
\text { plum/blackberry/Jaman) }\end{array}$ & $\begin{array}{l}\text { Syzygium cumini (L.) } \\
\text { Skeels }\end{array}$ & $\begin{array}{l}\text { Medium to large sized evergreen tree ascending to } \\
\text { about } 1500 \mathrm{~m} \text {, leaves are used for feeding cattle and } \\
\text { buffaloes during scarcity/lean period, seed is very } \\
\text { useful for diabetes, bark for fever, jaundice, } \\
\text { dysentery, urinary problems, sore-throats, } \\
\text { bronchitis, asthma, ulcers and chronic dysentery. }\end{array}$ \\
\hline $\begin{array}{l}\text { Khiang } \quad \text { (Needle } \\
\text { wood/Schima/Chilauni) }\end{array}$ & wallichii & $\begin{array}{l}\text { Large evergreen tree found up to } 1,500 \mathrm{~m} \text {, leaves } \\
\text { as fodders for cattle and buffaloes, fruits in powder } \\
\text { form is utilised as remedy for scorpion-sting, bites } \\
\text { of centipede and large black spider etc., juice of } \\
\text { bark is used as remedy for chronic ulcer and fresh } \\
\text { cuts. }\end{array}$ \\
\hline $\begin{array}{l}\text { Khawmhma (Chinese } \\
\text { sumac/nutgall tree) }\end{array}$ & Rhus chinensis Mill & $\begin{array}{l}\text { Small to medium sized deciduous tree ascending up } \\
\text { to } 700-1800 \mathrm{~m} \text {, leaves for feeding cattle and } \\
\text { buffaloes, decoction of fruits is recommended for } \\
\text { colic, diarrhoea and dysentery, leaves boiled in } \\
\text { water and the water is used for bath in suffering } \\
\text { from measles, juice of crushed leaves is applied to } \\
\text { rash or sores caused by Drimycarpus racemosus } \\
\text { and Semecarpus anacardium. }\end{array}$ \\
\hline Leh-ngo (Duggal fibre tree) & $\begin{array}{l}\text { Sarcochlamys } \\
\text { pulcherrima Gaudich }\end{array}$ & $\begin{array}{l}\text { Small evergreen tree, leaves used for feeding cattle, } \\
\text { buffaloes and pig, tender leaves are recommended } \\
\text { in diarrhoea and dysentery. }\end{array}$ \\
\hline $\begin{array}{l}\text { Lam-khuang/ } \\
\text { (Jackfruit tree/ Kathal) }\end{array}$ & $\begin{array}{l}\text { Artocarpus } \\
\text { heterophyllus Lam. }\end{array}$ & $\begin{array}{l}\text { Large evergreen tree found up to } 1200 \mathrm{~m} \text { above } \\
\text { MSL, leaves used for feeding small ruminants, } \\
\text { cattle and buffaloes, Decoction of the root is useful } \\
\text { in fever, diarrhoea, asthma, leaves for fever, skin } \\
\text { diseases, wounds, boils etc., milky juice of plant is } \\
\text { useful in sores caused by Herpes eruptions. }\end{array}$ \\
\hline Muk-fang (Indian cherry) & $\begin{array}{l}\text { Cordia dichotoma } \mathrm{G} . \\
\text { Forst }\end{array}$ & $\begin{array}{l}\text { Medium sized deciduous tree ascending to about } \\
1500 \mathrm{~m} \text {, leaves fed to cattle, buffaloes, small } \\
\text { ruminants. }\end{array}$ \\
\hline Ngiau (Champ) & $\begin{array}{l}\text { Magnolia roblonga } \\
\text { (Wall. ex Hook.f. \& } \\
\text { Thomson) Figlar }\end{array}$ & $\begin{array}{l}\text { Tall tree with oblanceolate leaves and white } \\
\text { flowers, leaves are utilised for cattle and buffaloes. }\end{array}$ \\
\hline $\begin{array}{l}\text { Nau-thak (Common grey } \\
\text { mango laurel/meda) }\end{array}$ & $\begin{array}{l}\text { Litsea monopetala } \\
\text { (Roxb.) Pers. }\end{array}$ & $\begin{array}{l}\text { Medium sized evergreen tree ascending to about } \\
1200 \mathrm{~m} \text {, leaves used for feeding cattle. }\end{array}$ \\
\hline Phan (Eastern elm) & $\begin{array}{l}\text { Ulmus lanceifolia } \\
\text { Roxb. Ex Wall }\end{array}$ & $\begin{array}{l}\text { Large deciduous tree ascending to about } 1400 \mathrm{~m} \text { in } \\
\text { Mizoram, leaves used for cattle buffaloes. }\end{array}$ \\
\hline $\begin{array}{ll}\text { Ri-hnim } & \text { (Kel- } \\
\text { hmawng/Hmawng-kir) }\end{array}$ & Indian Laurel Fig & $\begin{array}{l}\text { Medium to large sized evergreen tree found below } \\
1000 \mathrm{~m} \text { of MSL, leaves eaten by cattle, buffaloes, } \\
\text { goat and other ruminant species, juice of leaves is } \\
\text { used as remedy for flatulent colic and liver disease, } \\
\text { bark is used in rheumatism. }\end{array}$ \\
\hline Si-hneh & $\begin{array}{l}\text { Eurya cerasifolia } \\
\text { (D.Don) Kobuski }\end{array}$ & $\begin{array}{l}\text { Small evergreen tree found at } 900 \mathrm{~m} \text { above MSL, } \\
\text { leaves are lopped for cattle and buffaloes. }\end{array}$ \\
\hline Sa-zu-thei-pui (hairy fig) & Ficus hirta Vahl. & $\begin{array}{l}\text { Deciduous tree found up } 1500 \mathrm{~m} \text {, leaves used as } \\
\text { fodders for animals. }\end{array}$ \\
\hline Thing-kha & Derris robusta (DC.) & Medium sized deciduous tree found below $900 \mathrm{~m}$, \\
\hline
\end{tabular}




\begin{tabular}{|c|c|c|}
\hline & Benth. & $\begin{array}{l}\text { leaves are lopped for cattle; Decoction of the bark is } \\
\text { used as effective remedy for diabetes and high } \\
\text { blood pressure. }\end{array}$ \\
\hline Thel-ret (Thial-ret) & $\begin{array}{l}\text { Ficus elastica Roxb. } \\
\text { Ex Hoemen. }\end{array}$ & $\begin{array}{l}\text { Large evergreen tree with numerous aerial roots } \\
\text { found below } 1000 \mathrm{~m} \text {, leaves are good fodders. }\end{array}$ \\
\hline Thing-lung & $\begin{array}{l}\text { Homalium } \\
\text { (Gardner) reylanicum } \\
\text { Subsp. minutiflorum } \\
\text { (Kurz.) Mitra }\end{array}$ & $\begin{array}{l}\text { Evergreen tree with white flowers found up to } \\
1500 \mathrm{~m} \text { above MSL, leaves are utilised as fodders } \\
\text { during lean season. }\end{array}$ \\
\hline $\begin{array}{lr}\text { Tlai-zawng/ } & \text { Pai-vun } \\
\text { (Padam/wild } & \text { Himalayan } \\
\text { cherry) } & \\
\end{array}$ & $\begin{array}{l}\text { Cerasus cerasoides } \\
\text { (Buch.-Ham.exD.Don) } \\
\text { S.Y.Sokolov }\end{array}$ & $\begin{array}{l}\text { Medium sized tree found between altitudes } 700- \\
1800 \mathrm{~m} \text { above MSL, leaves are utilised as fodders } \\
\text { Decoction of the bark is used in fever. }\end{array}$ \\
\hline $\begin{array}{l}\text { Thing-hmar-cha (Hackberry/ } \\
\text { Stinwood) }\end{array}$ & Celtis timorensis Span. & $\begin{array}{l}\text { Medium sized evergreen tree found between } 800- \\
1500 \mathrm{~m} \text { leaves as fodders for cattle. }\end{array}$ \\
\hline $\begin{array}{ll}\text { Thei-tat } & \text { (Monkey } \\
\text { jack/Lakooch) } & \end{array}$ & $\begin{array}{l}\text { Artocarpus lakoocha } \\
\text { Roxb. }\end{array}$ & $\begin{array}{l}\text { Large deciduous tree found up to altitude } 1200 \mathrm{~m} \text {, } \\
\text { leaves as fodders for cattle. }\end{array}$ \\
\hline Thingsia (Chestnut) & $\begin{array}{l}\text { Castanopsis tribuloides } \\
\text { (Sm.) A.DC }\end{array}$ & $\begin{array}{l}\text { Medium to large sized evergreen tree ascending up } \\
\text { to latitude } 1800 \mathrm{~m} \text {, leaves used as fodders. }\end{array}$ \\
\hline Thing-ri (Black siris) & $\begin{array}{l}\text { Albizia odoratissima } \\
\text { (L.f.) Benth }\end{array}$ & $\begin{array}{l}\text { Medium sized deciduous tree found up to altitude } \\
1300 \mathrm{~m} \text {, leaves very good fodders for cattle, goat } \\
\text { and sheep. }\end{array}$ \\
\hline $\begin{array}{l}\text { Thei-ria } \\
\text { (Carallia/Corkwood) }\end{array}$ & $\begin{array}{l}\text { Carallia brachiata } \\
\text { (Lour.) Merr. }\end{array}$ & $\begin{array}{l}\text { Medium sized evergreen tree found up to altitude } \\
1200 \mathrm{~m} \text { leaves are lopped for fodders, bark and } \\
\text { leaves are used for septic poisoning and itching. }\end{array}$ \\
\hline Tiar & $\begin{array}{l}\text { Saurauia punduana } \\
\text { Wall }\end{array}$ & $\begin{array}{l}\text { Small evergreen tree, found ascending up to about } \\
1800 \mathrm{~m} \text { above MSL, leaves are utilised as fodders } \\
\text { for cattle and buffaloes. }\end{array}$ \\
\hline Thlan-vawng & Gmelina arborea Roxb & $\begin{array}{l}\text { Medium to large sized deciduous tree found } \\
\text { ascending up to } 1300 \mathrm{~m} \text {, leaves are liked by cattle } \\
\text { and hence used as fodders. }\end{array}$ \\
\hline Thing-vawk-pui & $\begin{array}{l}\text { Balakata baccata } \\
\text { (Roxb.) Esser }\end{array}$ & $\begin{array}{l}\text { Medium to large sized evergreen tree, leaves are } \\
\text { used as fodder for cattle and buffaloes. }\end{array}$ \\
\hline Thei-tit & $\begin{array}{l}\text { Ficus prostrate (Wall. } \\
\text { ex Miq.) Buch.-Ham. } \\
\text { ex Miq. }\end{array}$ & $\begin{array}{l}\text { Small deciduous tree found ascending up to about } \\
1500 \mathrm{~m} \text {, leaves for feeding to dairy cattle and } \\
\text { buffaloes, Juice of the root is recommended for } \\
\text { snake-bite and the white latex is applied on snake } \\
\text { bite. }\end{array}$ \\
\hline Vau-be & Bauhinia variegate $\mathrm{L}$. & $\begin{array}{l}\text { Medium sized deciduous tree found up to } 1500 \mathrm{~m} \text {, } \\
\text { leaves good fodders for the dairy animals, } \\
\text { Decoction of the bark or leaves is recommended for } \\
\text { menstrual disorders in female, intestinal worms, } \\
\text { piles, diabetes, diarrhoea and dysentery. }\end{array}$ \\
\hline Vang & $\begin{array}{l}\text { Albizia chinensis }(\mathrm{Osb}) \\
\text { Merr. }\end{array}$ & $\begin{array}{l}\text { Large deciduous tree found up to altitude about } \\
1200 \mathrm{~m} \text {, leaves good fodder for cattle, Infusion of } \\
\text { the bark is used as lotion for cuts, scabies and other } \\
\text { skin diseases. }\end{array}$ \\
\hline Zih-nghal & $\begin{array}{l}\text { Stereospermum } \\
\text { chelonoides (L.f.) DC. }\end{array}$ & $\begin{array}{l}\text { Medium sized tree (9-18 } \mathrm{m} \text { tall) found up to altitude } \\
1500 \mathrm{~m} \text {, leaves are very good fodder for cattle. }\end{array}$ \\
\hline Zai-rum (Yon) & $\begin{array}{l}\text { Anogeissus acuminate } \\
\text { (Roxb. Ex DC) Gull. }\end{array}$ & $\begin{array}{l}\text { Large deciduous tree found within about } 1000 \mathrm{~m} \\
\text { altitude, leaves are very good fodder for dairy } \\
\text { animals, decoction of the bark is recommended in } \\
\text { stomach troubles, fever, diarrhoea and also applied } \\
\text { on measles, chicken pox, sprains and burns, leaves } \\
\text { after cooking in water, the water is recommended as } \\
\text { remedy for high blood pressure. }\end{array}$ \\
\hline
\end{tabular}


Table.3 Total phenolics and Condensed tannins (CT) as leucocyanidin equivalent (\% on DM basis) in tree foliages fed to dairy animals in Mizoram

\begin{tabular}{|c|c|c|}
\hline Mizo name (Botanical Name) & $\begin{array}{c}\text { Total Phenolics }(\% \text { on } \\
\text { dry matter basis }) \\
(\mathrm{N}=6)\end{array}$ & $\begin{array}{l}\text { Condensed tannins }(\mathrm{CT}) \text { as } \\
\text { leucocyanidin equivalent }(\% \\
\text { on dry matter basis) }(\mathrm{N}=6)\end{array}$ \\
\hline An-ku/ An-ku-hawng-tial (Celtis tetrandra Roxb.) & $8.8607 \pm 0.74$ & $0.3762 \pm 0.12$ \\
\hline Bel-phuar [Trema orientalis (L.) Blume] & $7.4740 \pm 1.20$ & $0.3726 \pm 0.12$ \\
\hline Bung (Ficus benghalensis Linn.) & $6.7541 \pm 0.54$ & $0.1374 \pm 0.04$ \\
\hline $\begin{array}{l}\text { Ba-tling (Wendlandia budleioides Wall. Ex Wight \& } \\
\text { Arn.) }\end{array}$ & $25.5852 \pm 0.70$ & $3.9009 \pm 1.30$ \\
\hline Bil [Protium serratum (Wall. ex Colebr.) Engl.] & $23.1611 \pm 8.27$ & $5.9558 \pm 1.98$ \\
\hline Chawm-zil [Ligustrum robustum (Roxb.) Blume] & $18.3452 \pm 1.10$ & $0.3436 \pm 0.12$ \\
\hline $\begin{array}{l}\text { Chhawn-tual [Aporosa octandra (Buch.-Ham. ex D. } \\
\text { Don) Vickery] }\end{array}$ & $4.9477 \pm 0.78$ & $0.5700 \pm 0.26$ \\
\hline Hnum (Engelhardtia spicata Lechen ex Blume) & $13.8702 \pm 2.02$ & $0.5144 \pm 0.19$ \\
\hline Hnah-thap [Colona floribunda (Kurz) Craib] & $8.0950 \pm 0.98$ & $0.4486 \pm 0.15$ \\
\hline Hnahkiah (Callicarpa arborea Roxb.) & $12.7062 \pm 1.04$ & $0.3551 \pm 0.10$ \\
\hline Hriang (Betula alnoides Buch.-Ham. ex D. Don.) & $5.5323 \pm 0.47$ & $0.3644 \pm 0.13$ \\
\hline Hmui-pui/ Len-hmui [Syzygium cumini (L.) Skeels] & $22.4580 \pm 7.25$ & $7.0692 \pm 2.53$ \\
\hline Khiang (Schima wallichii Choisy) & $21.4894 \pm 5.43$ & $0.6156 \pm 0.21$ \\
\hline Khawmhma (Rhus chinensis Mill) & $26.7532 \pm 9.62$ & $1.8435 \pm 0.38$ \\
\hline Leh-ngo (Sarcochlamys pulcherrima Gaudich) & $4.8884 \pm 0.17$ & $0.1165 \pm 0.04$ \\
\hline Lam-khuang (Artocarpus heterophyllus Lam.) & $13.3478 \pm 1.01$ & $0.4242 \pm 0.15$ \\
\hline Muk-fang (Cordia dichotoma G. Forst) & $13.0928 \pm 1.45$ & $0.2653 \pm 0.07$ \\
\hline $\begin{array}{l}\text { Ngiau [Magnolia oblonga (Wall. ex Hook.f. \& } \\
\text { Thomson) Figlar] }\end{array}$ & $22.1938 \pm 5.89$ & $0.4366 \pm 0.15$ \\
\hline Nau-thak [Litsea monopetala (Roxb.) Pers.] & $4.6493 \pm 0.74$ & $1.1991 \pm 0.46$ \\
\hline Phan (Ulmus lanceifolia Roxb. Ex Wall.) & $7.2829 \pm 0.46$ & $1.2222 \pm 0.44$ \\
\hline Ri-hnim (Ficus retusa L.) & $32.5933 \pm 7.48$ & $3.4690 \pm 1.21$ \\
\hline Si-hneh [Eurya cerasifolia (D.Don) Kobuski] & $16.1235 \pm 1.25$ & $3.2251 \pm 1.03$ \\
\hline Sa-zu-thei-pui (Ficus hirta Vahl.) & $7.7894 \pm 1.11$ & $0.3322 \pm 0.11$ \\
\hline Thing-kha [Derris robusta (DC.) Benth.] & $12.1166 \pm 2.93$ & $0.8224 \pm 0.31$ \\
\hline Thel-ret [Ficus elastica Roxb. Ex Hoemen.] & $4.8824 \pm 0.81$ & $0.1139 \pm 0.04$ \\
\hline $\begin{array}{l}\text { Thing-lung [Homalium ceylanicum (Gardner) Benth. } \\
\text { Subsp. minutiflorum (Kurz.) Mitra] }\end{array}$ & $26.0483 \pm 1.37$ & $1.9656 \pm 0.66$ \\
\hline $\begin{array}{l}\text { Tlai-zawng/ Pai-vun [Cerasus cerasoides (Buch.- } \\
\text { Ham.exD.Don) S.Y.Sokolov] }\end{array}$ & $4.8908 \pm 0.64$ & $0.2114 \pm 0.07$ \\
\hline Thing-hmar-cha [Celtis timorensis Span.] & $9.7337 \pm 2.01$ & $0.5530 \pm 0.21$ \\
\hline Thei-tat (Artocarpus lakoocha Roxb.) & $27.8499 \pm 3.96$ & $1.0695 \pm 0.34$ \\
\hline Thingsia [Castanopsis tribuloides (Sm.) A.DC.] & $14.8031 \pm 2.85$ & $0.8352 \pm 0.31$ \\
\hline Thing-ri [Albizia odoratissima (L.f.) Benth.] & $36.9048 \pm 3.19$ & $0.9444 \pm 0.34$ \\
\hline Thei-ria [Carallia brachiata (Lour.) Merr.] & $4.3048 \pm 0.79$ & $3.7094 \pm 1.15$ \\
\hline Tiar (Saurauia punduana Wall.) & $7.1805 \pm 0.63$ & $0.5837 \pm 0.24$ \\
\hline Thlan-vawng [Gmelina arborea Roxb.] & $26.3336 \pm 2.42$ & $0.5331 \pm 0.23$ \\
\hline Thing-vawk-pui [Balakata baccata (Roxb.) Esser] & $42.4319 \pm 5.83$ & $1.4909 \pm 0.46$ \\
\hline $\begin{array}{l}\text { Thei-tit [Ficus prostrate (Wall. ex Miq.) Buch.-Ham. } \\
\text { ex Miq.] }\end{array}$ & $27.8499 \pm 3.96$ & $5.8235 \pm 1.96$ \\
\hline Vau-be [Bauhinia variegate L.] & $9.0975 \pm 1.31$ & $0.9553 \pm 0.34$ \\
\hline Vang [Albizia chinensis (Osb) Merr.] & $7.8747 \pm 0.81$ & $0.1824 \pm 0.05$ \\
\hline Zih-nghal [Stereospermum chelonoides (L.f.) DC.] & $61.5564 \pm 3.91$ & $3.9228 \pm 1.64$ \\
\hline $\begin{array}{l}\text { Zai-rum [Anogeissus acuminate (Roxb. Ex DC) } \\
\text { Gull.] }\end{array}$ & $61.1845 \pm 8.42$ & $0.5992 \pm 0.19$ \\
\hline
\end{tabular}


Table.4 Quantitative estimation of Flavonoids, saponins and Cumarin in tree leaves fed to dairy cattle in Mizoram

\begin{tabular}{|c|c|c|c|}
\hline Mizo name (Botanical Name) & $\begin{array}{c}\text { Flavonoids (mg } \\
\text { Rutin } \\
\text { Equivalents/g) } \\
\end{array}$ & $\begin{array}{c}\text { Saponins (mg } \\
\text { Diosgenin } \\
\text { equivalent/g) }\end{array}$ & $\begin{array}{c}\text { Coumarin (mg } \\
\text { coumarin } \\
\text { equivalent/g) }\end{array}$ \\
\hline An-ku/ An-ku-hawng-tial (Celtis tetrandra Roxb.) & ND & $30.4833 \pm 0.97$ & $5.0867 \pm 0.05$ \\
\hline Bel-phuar [Trema orientalis (L.) Blume] & $1.6933 \pm 0.02$ & $30.1100 \pm 0.64$ & $17.4667 \pm 0.50$ \\
\hline Bung (Ficus benghalensis Linn.) & $106.4767 \pm 1.12$ & $19.8600 \pm 0.45$ & $0.2467 \pm 0.09$ \\
\hline $\begin{array}{l}\text { Ba-tling (Wendlandia budleioides Wall. Ex Wight \& } \\
\text { Arn.) }\end{array}$ & $126.6033 \pm 2.15$ & $112.5033 \pm 2.15$ & $14.5400 \pm 0.32$ \\
\hline Bil [Protium serratum (Wall. ex Colebr.) Engl.] & $1.7300 \pm 0.09$ & $104.5333 \pm 0.55$ & $9.9000 \pm 0.24$ \\
\hline Chawm-zil [Ligustrum robustum (Roxb.) Blume] & $40.1933 \pm 1.10$ & $36.6933 \pm 1.63$ & $9.8433 \pm 0.39$ \\
\hline $\begin{array}{l}\text { Chhawn-tual [Aporosa octandra (Buch.-Ham. ex D. } \\
\text { Don) Vickery] }\end{array}$ & $74.6000 \pm 0.98$ & $21.9767 \pm 0.31$ & $10.2533 \pm 0.38$ \\
\hline Hnum (Engelhardtia spicata Lechen ex Blume) & $40.0967 \pm 0.16$ & $24.7033 \pm 0.55$ & $10.3267 \pm 0.33$ \\
\hline Hnah-thap [Colona floribunda (Kurz) Craib] & $1.0167 \pm 0.08$ & $32.6833 \pm 0.25$ & $2.1767 \pm 0.13$ \\
\hline Hnahkiah (Callicarpa arborea Roxb.) & $23.0600 \pm 0.10$ & $34.7567 \pm 0.45$ & $14.8967 \pm 0.31$ \\
\hline Hriang (Betula alnoides Buch.-Ham. ex D. Don.) & $28.0500 \pm 0.28$ & $59.0100 \pm 0.95$ & $13.0133 \pm 0.37$ \\
\hline Hmui-pui/ Len-hmui [Syzygium cumini (L.) Skeels] & $169.4167 \pm 0.63$ & $27.7333 \pm 0.66$ & $10.6300 \pm 0.38$ \\
\hline Khiang (Schima wallichii Choisy) & $66.4033 \pm 0.59$ & $57.2267 \pm 0.33$ & $18.9567 \pm 0.14$ \\
\hline Khawmhma (Rhus chinensis Mill) & $127.6100 \pm 0.62$ & $102.1367 \pm 0.89$ & $16.6300 \pm 0.61$ \\
\hline Leh-ngo (Sarcochlamys pulcherrima Gaudich) & $0.0500 \pm 0.00$ & $102.1367 \pm 0.26$ & $5.1400 \pm 0.13$ \\
\hline Lam-khuang (Artocarpus heterophyllus Lam.) & $24.8300 \pm 0.32$ & $27.8067 \pm 0.53$ & $4.0800 \pm 0.15$ \\
\hline Muk-fang (Cordia dichotoma G. Forst) & $10.9167 \pm 0.40$ & $27.8067 \pm 0.29$ & $1.7533 \pm 0.04$ \\
\hline $\begin{array}{l}\text { Ngiau [Magnolia oblonga (Wall. ex Hook.f. \& } \\
\text { Thomson) Figlar] }\end{array}$ & $178.4833 \pm 0.83$ & $75.6600 \pm 0.61$ & $12.0033 \pm 0.40$ \\
\hline Nau-thak [Litsea monopetala (Roxb.) Pers.] & $88.4767 \pm 0.66$ & $65.3633 \pm 0.55$ & $5.9867 \pm 0.25$ \\
\hline Phan (Ulmus lanceifolia Roxb. Ex Wall.) & ND & ND & $10.7700 \pm 0.57$ \\
\hline Ri-hnim (Ficus retusa L.) & $115.3067 \pm 0.55$ & $97.8700 \pm 0.95$ & $6.1867 \pm 0.18$ \\
\hline Si-hneh [Eurya cerasifolia (D.Don) Kobuski] & $77.5467 \pm 0.56$ & $112.0667 \pm 1.24$ & $19.3700 \pm 0.74$ \\
\hline Sa-zu-thei-pui (Ficus hirta Vahl.) & $8.2300 \pm 0.22$ & $19.7000 \pm 0.48$ & $1.8467 \pm 0.04$ \\
\hline Thing-kha [Derris robusta (DC.) Benth.] & $60.0267 \pm 0.68$ & $44.7333 \pm 0.65$ & $19.7367 \pm 0.58$ \\
\hline Thel-ret [Ficus elastica Roxb. Ex Hoemen.] & $5.0767 \pm 0.10$ & $10.0667 \pm 0.33$ & $2.1767 \pm 0.11$ \\
\hline $\begin{array}{l}\text { Thing-lung [Homalium ceylanicum (Gardner) Benth. } \\
\text { Subsp. minutiflorum (Kurz.) Mitra] }\end{array}$ & $152.4233 \pm 1.09$ & $98.4733 \pm 0.60$ & $9.1667 \pm 0.19$ \\
\hline $\begin{array}{l}\text { Tlai-zawng/ Pai-vun [Cerasus cerasoides (Buch.- } \\
\text { Ham.exD.Don) S.Y.Sokolov] }\end{array}$ & $28.5267 \pm 0.48$ & $99.3067 \pm 0.49$ & $31.3833 \pm 0.71$ \\
\hline Thing-hmar-cha [Celtis timorensis Span.] & $27.9633 \pm 0.36$ & $37.4800 \pm 0.62$ & $14.5000 \pm 0.25$ \\
\hline Thei-tat (Artocarpus lakoocha Roxb.) & $15.1667 \pm 0.18$ & $79.5033 \pm 0.50$ & $3.4467 \pm 0.25$ \\
\hline Thingsia [Castanopsis tribuloides (Sm.) A.DC.] & $51.6833 \pm 0.54$ & $65.2067 \pm 0.29$ & $11.8500 \pm 0.25$ \\
\hline Thing-ri [Albizia odoratissima (L.f.) Benth.] & $7.0467 \pm 0.09$ & $99.0500 \pm 0.69$ & $5.2933 \pm 0.06$ \\
\hline Thei-ria [Carallia brachiata (Lour.) Merr.] & $54.2667 \pm 0.50$ & $95.6100 \pm 0.76$ & $7.2100 \pm 0.17$ \\
\hline Tiar (Saurauia punduana Wall.) & $17.1767 \pm 0.19$ & $37.0033 \pm 0.28$ & $5.1267 \pm 0.12$ \\
\hline Thlan-vawng [Gmelina arborea Roxb.] & $15.2167 \pm 0.16$ & $85.9233 \pm 0.53$ & $12.0967 \pm 0.48$ \\
\hline Thing-vawk-pui [Balakata baccata (Roxb.) Esser] & $130.1133 \pm 0.77$ & $93.5400 \pm 0.72$ & $28.0733 \pm 0.81$ \\
\hline $\begin{array}{l}\text { Thei-tit [Ficus prostrate (Wall. ex Miq.) Buch.-Ham. } \\
\text { ex Miq.] }\end{array}$ & ND & ND & $10.9533 \pm 0.51$ \\
\hline Vau-be [Bauhinia variegate L.] & $39.4367 \pm 0.49$ & $33.8767 \pm 0.37$ & $3.2367 \pm 0.08$ \\
\hline Vang [Albizia chinensis (Osb) Merr.] & $12.9767 \pm 0.47$ & $5.8200 \pm 0.18$ & $18.2600 \pm 0.38$ \\
\hline Zih-nghal [Stereospermum chelonoides (L.f.) DC.] & $18.2733 \pm 0.22$ & $102.1167 \pm 0.87$ & $5.9433 \pm 0.05$ \\
\hline $\begin{array}{l}\text { Zai-rum [Anogeissus acuminate (Roxb. Ex DC) } \\
\text { Gull.] }\end{array}$ & $119.6033 \pm 0.59$ & $30.3433 \pm 0.79$ & $19.6467 \pm 0.74$ \\
\hline
\end{tabular}


Table.5 Quantitative estimation of alkaloids in tree leaves fed to dairy cattle in Mizoram

\begin{tabular}{|l|c|}
\hline Name & Alkaloids (mg/g) \\
\hline Hnah-thap [Colona floribunda (Kurz) Craib] & $0.45 \pm 0.00$ \\
\hline Hriang (Betula alnoides Buch.-Ham. ex D. Don.) & $3.03 \pm 0.03$ \\
\hline Lam-khuang (Artocarpus heterophyllus Lam.) & $1.80 \pm 0.01$ \\
\hline Muk-fang (Cordia dichotoma G. Forst) & $0.18 \pm 0.01$ \\
\hline Ngiau [Magnolia oblonga (Wall. ex Hook.f. \& Thomson) Figlar] & $1.47 \pm 0.01$ \\
\hline Sa-zu-thei-pui (Ficus hirta Vahl.) & $0.86 \pm 0.01$ \\
\hline Thing-lung [Homalium ceylanicum (Gardner) Benth. Subsp. minutiflorum (Kurz.) Mitra] & $1.49 \pm 0.01$ \\
\hline Vau-be [Bauhinia variegate L.] & $0.80 \pm 0.00$ \\
\hline Zai-rum [Anogeissus acuminate (Roxb. Ex DC) Gull.] & $7.28 \pm 0.01$ \\
\hline
\end{tabular}

\section{Quantitative analysis of phytochemicals}

The DM\% of the tree leaves was varied from $16.04-22.95 \%$.

The total phenolics concentration was highly variable in different tree foliages varying from $4.3048 \pm 0.79 \%$ to $61.5564 \pm 3.91 \%$ on dry matter basis. The CT levels were also variable and ranged from $0.1165 \pm 0.04 \%$ to $7.0692 \pm 2.53 \%$ on dry matter basis (Table 3 ). New leaves often have higher tannin content than older leaves. In the present study, since leaves of various developmental stages were collected and mixed together for preparation of representative sample, the total phenolic content might be higher than the reported earlier.

Flavonoids and saponins were not detected in aqueous extracts of Ulmus lanceifolia Roxb. Ex Wall.(Phan) and Ficus prostrate (Wall. ex Miq.) Buch.-Ham. ex Miq. (Theitit); whereas flavonoids was not detected in Celtis tetrandra Roxb. (Anku). Leaves of other tree species had good concentrations of both flavonoids and saponins. Coumarin was detected in all the tree species. The flavonoids ranged from $0.0500 \pm 0.00$ to $178.4833 \pm 0.83$ mg RE/g dry sample, whereas saponins varied from $10.0667 \pm 0.33$ to $112.5033 \pm 2.15 \mathrm{mg}$ DE/g dry sample (Table 4). Highest level of coumarin was estimated in Cerasus cerasoides (Buch.-Ham.exD.Don) S.Y.Sokolov (31.3833 $\pm 0.71 \mathrm{mg}$ coumarin equivalent/g dry sample) and lowest level in Ficus benghalensis Linn $(0.2467 \pm 0.09 \mathrm{mg}$ coumarin equivalent/g dry sample). Kokila $e t$ al., (2013) reported that leaves of various species of Albizia contained saponins, terpenes, alkaloids and lavonoids. Lalawmpuii et al., (2014) estimated the total phenolics and flavonoids levels as $62.75 \mathrm{mg}$ GAE/g dry weight and $56 \mathrm{mg}$ QE/g dry weight respectively in Helicia nilagirica leaves, commonly known as 'Pasaltakaza' in Mizoram which is used for stomach ailments like peptic ulcer and indigestion by the people in Mizoram. Brighente et al., (2007) also reported the total phenolics and flavonoids level as $82.98 \pm 1.72 \mathrm{mg}$ GAE/g and $13.09 \pm 0.39 \mathrm{mg}$ QE/g (DM basis) in aqueous extract of Cyathea phalerata. Joseph and Raj (2011) also confirmed the presence of flavonoids in Ficus benghalensis Linn.

The alkaloids varied from $0.18 \pm 0.01 \mathrm{mg} / \mathrm{g}$ in Cordia dichotoma G. Forst (Muk-fang) to $7.28 \pm 0.01 \mathrm{mg} / \mathrm{g}$ in Anogeissus acuminate (Roxb. Ex DC) Gull. (Zai-rum) (Table 5). Devanaboyina et al., (2013) reported alkaloids in methanolic extract of Alstonia macrophylla as $42.69 \mu \mathrm{g}$ atropine equivalent $/ \mathrm{ml}$. Abidemi (2013) reported alkaloids level ranging from $80-533 \mathrm{mg} / 100 \mathrm{mg}$ in 7 selected medicinal plants, namely B. Vulgaris, E. hirta, $L$. Inarmic, M. Pudica, B. Pilosa, C. Zambesicus and $P$. americana in Nigeria. Sutharsingh et al., (2011) estimated total alkaloids level as $0.86 \pm 0.023 \%$ (w/w) in Naravalia zeylanica 
DC. Variability in total alkaloids level in different tree species of the present study thus in agreement with the variable concentrations of alkaloids reported earlier in different plant species by various workers.

It was concluded that the leaves of various tree species are rich sources of phytochemicals and possess significant antioxidant properties. Farmers of Mizoram have been utilizing these tree leaves without any scientific considerations from time immemorial. The baseline data gained through this study about content and concentration of phytochemicals and antioxidant property will help in their further study about usefulness as herbs and also include in total mixed ration (TMR) of dairy animals based on these properties and nutritional contents.

\section{Acknowledgement}

The author is obliged to Central Agricultural University, Imphal, Manipur, India for financial support and providing the facilities to carry out the study in the form of Intramural Research Project (CAU-DR/32(Vety)/2010/Vol.II/1072) of the University and to the Department of Forestry, Mizoram University, Aizawl, Mizoram for rendering help in identification of the species.

\section{References}

Abidemi, O.O., 2013. Phytochemicals and spectrophotometric determination of meals in various medicinal plants in Nigeria. International Journal of Engineering Science Invention. 2(5): 51-4.

Akenga, T., F.O. Orech, J. Ochora, H. Friis and Aagaard, H. 2005. Potential Toxicity of some Traditional Leafy Vegetables consumed in Nyangoma Division, Western Kenya. African Journal of Food and Nutritional Science. 5(1): 1-30.

Brighente, I.M.C., M. Dias, L.G. Verdi and Pizzolatti, M.G. 2007. Antioxidant activity and total phenolic content of some Brazilian species. Pharmaceutical Biology. 45(2): 156-161.

Das, K.C., S. Malik and Subudhi, P.K. 2006. Chemical composition of tree leaves and shrubs used as fodder in Mizoram. Indian Journal of Animal Sciences. 76(2): 163164.

Devanaboyina, N., N. Ramalakshmi, B. Satyanarayana, P. Sudeepthi, K. Hemachakradhar and Pavankumar Raju, N. 2013. Preliminary phytochemical screening, quantitative estimation and evaluation of antimicrobial activity of Alstonia macrophylla stem bark. Science Inventions Today. 2(1): 31-39.

Dinda, B., S. Debnath and Harigaya, Y. 2007. Naturally occurring iridoids - A review (Part 1). Chemical and Pharmaceutical Bulletin. 55: 159-222.

Diwan, P., A. Yashashree and Gadhikar. 2014. Phytochemical composition and inhibition of oral pathogens by ficus benghalensis (linn.) Root extracts. International Journal of Pharmacy and Pharmaceutical Sciences. 6(3):111-114.

Economic Survey Mizoram. 2013-14. Published by Planning \& Programme Implementation Department, Government of Mizoram, 2013-14.

Edeoga, H.O., D.E. Okwu and Mbaebie, B.O. 2005. Phytochemical Constituents of some Nigeria Medicinal Plants. African Journal of Biotechnology. 4(7): 685-688.

Egharevba, Henry Omoregie, Kunle and Folashade Oluyemisi. 2010. Preliminary Phytochemical and Proximate Analysis of the leaves of Piliostigma thonningii (Schumach.) Milne-Redhead. Ethnobotanical Leaflets. 14: 570-77.

Ganatra Sunil, H., P. Durge Shweta and Patil, S.U. 2012. Preliminary Phytochemicals Investigation and TLC Analysis of Ficus racemosa Leaves. J. Chem. Pharm. Res. 4(5): 2380-2384.

Joseph, Baby, and Raj, S. Justin. 2011. An overview- ficus bengalensis linn. International Journal of Pharmaceutical Sciences Review and Research. 6(1): 21-24.

Kaur, C., and Kapoor, H.C.2002. Anti-oxidant activity and total phenolic content of some 
Asian vegetables. International Journal of Food Science and Technology. 37: 153161.

Kokila, K., D.S. Priyadharshini and Venugopal, S. 2013. Phytopharmacological properties of Albizia species: A Review. International Journal of Pharmacy and Pharmaceutical Sciences. 5(3): 70-73.

Kumaresan, A., K.M. Bujarbaruah, K.A. Pathak and Ramesh, B.T. Soil-plant-animal continuum in relation to macro and micro mineral status of dairy cattle in subtropical hill agro ecosystem. Trop Anim Health Prod.42: 569-577.

Lalawmpuii, P.C., C. Malsawmtluangi, Zothanpuia and Kakoti, B.B. 2014. Phytochemical screening and determination of antioxidant activity of Helicia Nilagirica Bedd., an ethnomedical plant of Mizoram, India. Asian Journal of Pharmaceutical and Clinical Research. 7(4): 246-249.

Makkar, H.P.S., M. Bluemmel, N.K. Borowy and Becker, K. 1993. Gravimetric determination of tannins and their correlations with chemical and protein precipitation methods. J. Sci. Food Agric. 61:161-165.

Makkar, H.P.S., P. Siddhuraju and Becker, K. 2007. Methods in molecular biology: Plant secondary metabolites, Humana Press Inc., Totowa, New Jersey.

Nabavi, S.M., M.A. Ebrahimzadeh, S.F. Nabavi and Jafari, M.2008. Free radical scavenging activity and antioxidant capacity of eryngium caucasicum trautv and froripia subpinnata. Pharmacologyonline. 3: 19-25.

Podsędek, A., 2007. Natural antioxidants and antioxidant activity of Brassica vegetables: a review. LWT- Food Science and Technology. 40: 1-11.

Porter, L.J., L.N. Hrstich and Chan, B.G. The conversion of procyanidins and prodelphinidins to cyaniding and delphinidin. Phytochemistry. 25: 223-230.

Samanta, A.K., R. Buragohain, R.J. Kukde and Pawar, S. 2009. Nutritional evaluation of some common tree leaves of Aizawl district of Mizoram, In: Proceedings of Animal Nutrition Association World Conference, February 14-17, pp. 193.

Sarma, K., G. Kalita, C.N. Galdhar, K.C. Das, B. Saikia, R. Buragohain and Borthakur, S.K. 2007. Mineral status of forest fodder in Aizawl district of Mizoram. Indian J. Animal Sciences. 77(11): 1199-1200.

Sofowora, A., 1982. Medicinal Plants and Traditional medicine in Africa, John Wiley and Sons.

Sowjanya, K.M., J. Swathi, K. Narendra and Krishna Satya, A. 2013. A review on phytochemical constituents and bioassay of syzygium cumini. International Journal of Natural Product Science. 3(2):1-11.

Sutharsingh, R., S. Ravimani, B. Jayakar, M. Uvarani and Thangathirupathi, A. 2011. Quantitative phytochemical estimation and antioxidant studies on aerial parts of Naravalia zeylanica DC. International Journal of Pharmaceutical Studies and Research. 2(2): 52-56.

Trease, G.E., and Evans, W.C. 1989. Pharmacognsy, 11th edition, Brilliar Tiridel can. Macmillian Publishers.

Uusiku, N.P., A. Oelofse, K.G. Duodu, M.J. Bester and Faber, M. 2010. Nutritional value of leafy vegetables of sub-Saharan Africa and their potential contribution to human health: a review. Journal of Food Composition and Analysis. 23: 499-509.

\section{How to cite this article:}

Rajat Buragohain. 2017. Tree Foliages Fed to Dairy Animals in Mizoram: Traditional Medicinal Uses, Screening and Quantification of Phytochemicals. Int.J.Curr.Microbiol.App.Sci. 6(10): 61-71. doi: https://doi.org/10.20546/ijcmas.2017.610.008 